\title{
Nonisothermal homogeneous nucleation
}

\author{
B. E. Wyslouzil and J. H. Seinfeld \\ Department of Chemical Engineering, California Institute of Technology, Pasadena, California 91125
}

(Received 2 August 1991; accepted 28 April 1992)

\begin{abstract}
Classical homogeneous nucleation theory is extended to nonisothermal conditions through simultaneous cluster mass and energy balances. The transient nucleation of water vapor following a sudden increase in saturation ratio is studied by numerically solving the coupled mass and energy balance equations. The ultimate steady state nucleation rate, considering nonisothermal effects, is found to be lower than the corresponding isothermal rate, with the discrepancy increasing as the pressure of the background gas decreases.

After the decay of the initial temperature transients, subcritical clusters in the vicinity of the critical cluster are found to have temperatures elevated with respect to that of the background gas.
\end{abstract}

\section{INTRODUCTION}

Classical homogeneous nucleation theory provides a simple physical model that describes the formation of a new phase from a supersaturated phase in the absence of foreign seed particles. In this model the new phase appears by the growth of clusters by monomer addition and, in the case of the gas to liquid phase transition, each cluster is assumed to behave as if it were an incompressible liquid drop with the physical properties of the bulk material. In addition all of the clusters and the monomer are assumed to be at the same temperature. Using these assumptions it is possible to solve for the steady state, isothermal rate of formation of stable particles of the new phase as a function of the bulk physical properties of the material, the temperature, and the degree of supersaturation of the system. ${ }^{1,2}$ By assuming isothermal conditions classical nucleation theory ignores any of the processes that could contribute to temperature differences between the old and the new phases, in particular any energy released or required by the phase transition. This, in turn, implies that the effect of nonisothermal conditions on the mass balances used to determine the rate of formation of the new phase is also ignored. The theory effectively separates the nucleation process from any potential effects that could influence the transfer of energy to and from the clusters, such as the amount and chemical nature of any background gas present.

That all of the clusters of a given size in a nucleating system will have the same temperature or energy is clearly an approximation. If the liquid drop model is adopted, the energy of the cluster is a well defined quantity equal to the product of the number of monomers in the cluster and the bulk energy per molecule of the new phase at the temperature of the cluster. In the gas to liquid phase transition, for example, the latent heat that accompanies a condensing monomcr brings more energy to the growing cluster than would be contributed by the addition of another liquidlike monomer. Thus clusters that have just been formed by the addition of a monomer will have on average more energy and be on average warmer than those which have just been formed by evaporation, with clusters at a whole range of temperatures in between depending on the number of collisions that have occurred with background gas molecules since a particular cluster last gained or lost a monomer. The warmer clusters will exhibit a higher evaporation rate than the cooler clusters and hence the average evaporation rate of clusters of a given size will depend on the relative populations of hot and cold clusters and their respective evaporation rates. The processes that lead to distributions in temperature within a given cluster size class exist whether the system on the whole is in a transient state or at steady state. However, at steady state a change in the relative rates of evaporation and condensation due to nonisothermal effects, over those predicted by the classical theory, will naturally alter the overall rate of formation of the new phase.

While there is the potential for a distribution of temperatures within a given cluster size, the average temperature of each cluster size will also be determined by a balance between the relative rates at which clusters are formed by condensation and evaporation vs the rate at which energy can be removed by collisions with nonaccommodated monomers or inert background gas molecules. Again, this is true whether the total number of clusters of a given size is changing or not. Thus the clusters containing $g$ monomers will be characterized by a temperature distribution function, $p_{g}\left(T_{g}\right)$, whose mean is $\bar{T}_{g}$. If the rate of energy transfer by collisions is very high because of a sufficient amount of background gas or a low mass accommodation of the nucleating species, it is reasonable to assume that $T_{g}$ (dropping the overbar notation) will be very close to $T_{\mathrm{amb}}$, the temperature of the ambient background gas. However, the width of the distribution $p_{g}\left(T_{g}\right)$ will still be nonzero simply because there are distributions of energy in the colliding and condensing molecules themselves.

Both experimental and theoretical evidence exists demonstrating that the incorporation of a vapor monomer into a cluster has the potential to raise the temperature of the cluster significantly. During the nucleation and growth of iron clusters from the vapor phase in high temperature shock tube experiments, Kung and Bauer ${ }^{3}$ used the ratio of emission intensities at two different wavelengths to estimate the nucleated particle temperatures and observed that 
the black body temperature of the clusters was significantly higher than that of the background gas. This positive temperature difference persisted as long as condensation dominated. In simulations of the addition of a monomer to water pentamer clusters, Plummer and $\mathrm{Chen}^{4}$ found that at an initial temperature of $93 \mathrm{~K}$ the water monomer was incorporated and resulted in a hexamer with a final temperature of 174 or $184 \mathrm{~K}$ depending on the final configuration. As cluster size increases the effect that incorporating a monomer into a cluster has on the final temperature of the cluster should decrease because the total heat capacity of the cluster increases.

Aspects of nonisothermal homogeneous nucleation have been addressed previously by Kantrowitz, ${ }^{5}$ Feder et $a l .{ }^{6}{ }^{\text {Salpeter, }}{ }^{7}$ Grinin and Kuni, ${ }^{8}$ and Ford and Clement. ${ }^{9}$ Kantrowitz ${ }^{5}$ used a simple energy balance to evaluate the average steady state temperature of the clusters assuming that all of the clusters are at the same elevated temperature, i.e., $T_{g}=T_{g+1}=T_{g+2}=\cdots$. From this he was able to predict an adjustment to the isothermal nucleation rate for small temperature differences. The assumption that all of the clusters are at the same temperature and that this is the temperature of the critical cluster does not consider the behavior of smaller clusters where the effect of monomer addition is more important. Moreover, since critical clusters result from the growth of smaller clusters, hindered growth of subcritical clusters may affect the final concentration of stable clusters, slowing the nucleation process in a manner that is not apparent simply by focusing on clusters in the critical region. The analysis of nonisothermal effects in nucleation by Feder et al..$^{6}$ included the effect of fluctuations in the energy of the clusters on the steady state nucleation rate. The authors found only a small change in the nucleation rate with respect to the isothermal theory, largely because the critical cluster is both in thermal and chemical equilibrium with the surrounding vapor. Consequently, when calculating the nucleation rate based on an analysis in the region of the critical nucleus, the concentration of critical clusters is unperturbed from that predicted by isothermal theory and the reduction in nucleation rate results solely from the nonisothermal diffusion coefficient. Again, the analysis is limited to the behavior of clusters in the critical region.

In the present work we wish to develop a theory of nonisothermal homogeneous nucleation. An energy balance applied to each cluster size, analogous to the mass balance, provides the most straightforward method of incorporating the strong coupling that exists between the mass and energy flows due to the evaporation rate coefficient. Because Feder et al. ${ }^{6}$ showed that the effects of energy fluctuations within a cluster size class on the nucleation rate are rather small, these are ignored and only the average energy of all clusters of a given size is followed.

The objective of the current work is to demonstrate how the relative rates of mass and energy transfer combine to determine both the transient and steady state cluster concentrations, cluster temperature distributions, and mass and energy fluxes for both subcritical and stable clusters. By simultaneously solving the differential equations gov- erning the number and enthalpy evolution of the clusters as the supersaturation is suddenly increased from 1.0 to a constant higher value, both the transient and steady state behavior of the average cluster concentration and energy may be followed and compared with the isothermal results. The role of the mass accommodation coefficient as a factor in the energy transfer process, especially as the concentration of background gas decreases, is also investigated because it provides an alternate mechanism to change the relative rates of energy and mass transfer. The nature of energy transfer to the subcritical clusters is discussed in terms of a model for energy transfer between excited state and background gas molecules from unimolecular reaction theory which in turn demonstrates how the chemical nature of both the nucleating species and the background gas can play a role in the homogeneous nucleation process. In order to compare the results of these nucleation rate calculations to previously published isothermal computations, ${ }^{10,11}$ the vapor to liquid phase transition of water is studied.

\section{CLUSTER MASS AND ENERGY BALANCES}

A model for the formation of a new phase from a supersaturated parent phase should address the mechanism of incorporation of individual molecules into the emerging clusters and the redistribution of energy that occurs by the given phase transition. For the special case of the vapor to liquid phase transition the change in energy upon condensation, $\Delta h_{\text {vap }}=h_{\text {vapor }}-h_{\text {liquid, }}$ is always positive, but in other processes, such as crystallization, $\Delta h_{\text {phase } 1 \rightarrow \text { phase } 2}$ can have either sign. Thus the incorporation or loss of a monomer from a cluster is always accompanied by a change in energy of the order of $q=\Delta h_{\text {vap }}-w$, where $w$ is the work required to increase the area of the interface. Collisions with inert, background gas molecules or monomers that are not accommodated provide an alternative means of adding or removing energy from the cluster and will on average reduce the temperature differences between the new phase and the background gas resulting from the addition or loss of condensing molecules. Because the rate at which monomers condense or evaporate from the cluster may be a strong function of the temperature of a cluster, it is natural to try and understand how the simultaneous transfer of mass and energy affects the transient and final steady state rates with which this phase transition occurs.

Implicit in the formultion of the problem presented here is the assumption that all of the clusters of a given size are characterized by a mean temperature, $T_{g}$. This assumption will capture the essential physics of the strong coupling between the mass and energy fluxes that results from the temperature dependent evaporation rate coefficient. We also assume that the temperature of the background gas and the condensing monomer are characterized by a fixed ambient temperature, $T_{\text {amb }}$.

\section{A. Cluster mass balance}

To develop the cluster mass balance we assume that clusters of size $g$ have concentration $f_{g}$ and grow by the addition of monomer at a rate $\beta_{g} f_{g}$ or shrink by the loss of 
a monomer at a rate $\alpha_{g} f_{g}$, where $\beta_{g}$ and $\alpha_{g}$ are the condensation and evaporation rate coefficients, respectively. The overall flux of clusters from $g-1$ to $g$ is given by

$$
I_{g}=\beta_{g-1} f_{g-1}-\alpha_{g} f_{g}
$$

As in classical nucleation theory only growth by loss or addition of monomers is considered. The evolution of the $g$-mer cluster concentration, $f_{g}$, is therefore given by

$$
\frac{d f_{g}}{d t}=I_{g}-I_{g+1} \text {. }
$$

The kinetic gas expression is used for the impingement rate of monomer onto the cluster containing $g$ molecules, $\beta_{g}$, and is given by

$$
\beta_{g}=A_{g} \frac{p}{\left(2 \pi m k T_{\text {amb }}\right)^{1 / 2}},
$$

where $A_{g}$ is the surface area of the $g$-mer, $p$ is the partial pressure of the nuclcating vapor, $m$ is the molecular mass of the vapor, $k$ is the Boltzmann constant, and $T_{\text {amb }}$ is the absolute ambient temperature. Assuming, as in classical nucleation theory, that each cluster behaves as if it were an incompressible liquid drop with the same physical properties as the bulk material, $A_{g}=4 \pi\left(3 v_{m} / 4 \pi\right)^{2 / 3} g^{2 / 3}$, where $v_{m}$ is the molecular volume.

The evaporation coefficient from clusters containing $g+1$ molecules, $\alpha_{g+1}$, is a function only of the properties of the cluster itself and is calculated from the detailed balance condition of the equilibrium cluster distribution at the temperature $T$ and saturation ratio $S$,

$$
\begin{aligned}
\alpha_{g+1} & =\beta_{g} \frac{N_{g}^{e}}{N_{g+1}^{e}} \\
& =\beta_{g} \exp \left\{-\ln S+\frac{\sigma A_{1}}{k T}\left[(g+1)^{2 / 3}-g^{2 / 3}\right]\right\} \\
& =A_{g} \frac{p_{\mathrm{ve}}(T)}{(2 \pi m k T)^{1 / 2}} \exp \left\{\frac{\sigma A_{1}}{k T}\left[(g+1)^{2 / 3}-g^{2 / 3}\right]\right\},
\end{aligned}
$$

where $N_{g}^{e}$ is the equilibrium cluster concentration of $g$ mers, $\sigma$ is the surface tension, and $A_{1}$ is the surface area per molecule $\left[=4 \pi\left(3 v_{m} / 4 \pi\right)^{2 / 3}\right]$, and $p_{\mathrm{ve}}(T)$ is the equilibrium vapor pressure over a flat surface at $T$.

Equations (1)-(4) are those that $\mathrm{Abraham}^{10}$ and Courtney ${ }^{11}$ first solved numerically in order to gain a better understanding of the transient kinetics and time lags involved as an isothermal system relaxes to the steady state.

\section{B. Cluster energy balance}

To estimate the transient flow of energy through the system of clusters as well as at the final steady state an energy balance including the effects of condensation, evaporation, and heat transfer by impinging but noncondensing molecules has been derived by considering the relevant energy fluxes. The rate of change of the total enthalpy of the $g$-mers, $h_{g} f_{g}$ is governed by the following: (1) the rate at which energy enters the class of $g$-mers by the formation of new $g$-mers by condensation of a monomer onto $g$-1-mers or by monomer evaporation from $g+1$-mers, (2) the rate at which energy leaves the class of $g$-mers by the growth or evaporation of $g$-mers, and (3) the rate at which energy is removed from the $g$-mers by collisions with the background gas or noncondensing monomers. In addition, when the system is in equilibrium at $S=1$, the clusters should all be characterized by the same temperature $T_{g-1}=T_{g}=T_{g+1}=T_{\text {amb. }}$.

With the assumption of the liquid drop model for the cluster, the enthalpy of a single $g$-mer at its average temperature $T_{g}$ is calculated as

$$
h_{g}=h_{g}^{0}+g c_{p}\left(T_{g}-T_{\mathrm{ref}}\right) \text {, }
$$

which simplifies when $h_{g}^{0}=0$ at $T=T_{\text {ref }}$ and where $c_{p}$ is the liquid heat capacity per molecule. This equation also defines the average temperature of a $g$-mer from the values of total enthalpy and cluster concentration as

$$
T_{g}=T_{\text {ref }}+\frac{h_{g} f_{g}}{g c_{p} f_{g}}
$$

The equilibrium constraint at $S=1$ and $T=T_{e}$ determines the amount of energy removed from the class of $g$-mers each time a $g$-mer evaporates. If $q_{g-1}$ is the energy released per monomer incorporated into a growing cluster initially of size $g-1$, the rate at which energy enters the class of $g$-mers by this condensation process is simply given by $\beta_{g-1} f_{g-1}\left[h_{g-1}\left(T_{g-1}\right)+q_{g-1}\right]$. This expression and the equilibrium constraint at $S=1$ and $T=T_{e}$ then determine the rate of energy removal from the class of $g$-mers when evaporation of monomers from the $g$-mers forms $g$-1-mers. At equilibrium the following energy balance must hold between adjacent cluster sizes to maintain all of the clusters at the average temperature of the system $T_{e}$ :

$$
\beta_{g-1} f_{g-1}\left[h_{g-1}\left(T_{e}\right)+q_{g-1}\right]=\alpha_{g} f_{g}\left[h_{g}\left(T_{e}\right)+\Delta\right],
$$

where $\Delta=q_{g-1}-h_{1}\left(T_{e}\right)$. This balance simply states that at equilibrium the net energy flux between adjacent cluster sizes is zero and by inspection Eq. (7) implies that $\Delta$ $=q_{g-1}-h_{1}\left(T_{e}\right)$ where $h_{1}\left(T_{e}\right)=h_{g}\left(T_{e}\right)-h_{g-1}\left(T_{e}\right)$ $=1 \cdot C_{p}\left(T_{e}-T_{\text {ref }}\right)$. Generalizing Eq. (7) to an arbitrary temperature $T_{g}$ gives the rate at which energy is lost from the class of $g$-mers by evaporation as

$$
\alpha_{g} f_{g}\left[h_{g}\left(T_{g}\right)+\Delta\right]=\alpha_{g} f_{g}\left[h_{g-1}\left(T_{g}\right)+q_{g-1}\right] .
$$

Thus the overall transient energy balance on the class of $g$-mers becomes

$$
\begin{aligned}
\frac{d\left(h_{g} f_{g}\right)}{d t}= & \beta_{g-1} f_{g-1}\left[h_{g-1}\left(T_{g-1}\right)+q_{g-1}\right] \\
& -\alpha_{g} f_{g}\left[h_{g-1}\left(T_{g}\right)+q_{g-1}\right]-\beta_{g} h_{g} f_{g} \\
& +\alpha_{g+1} f_{g+1}\left[h_{g}\left(T_{g+1}\right)\right]-Q_{g}
\end{aligned}
$$

where $Q_{g}$ is the rate at which energy is removed from the class of $g$-mers by collisions with background gas or noncondensing monomers. Clearly this energy balance satisfies the condition that at $S=1, T_{g-1}=T_{g}=T_{g+1}=T_{\text {amb }}$.

A key quantity in the energy balance is $q_{g}$, the energy released per monomer incorporated into the growing clus- 
ter. In the liquid drop model this quantity is equal to the heat of condensation per molecule, $h_{\mathrm{vap}}^{\infty}=\Delta H_{\mathrm{vap}}^{\infty} / N_{A}$, where $\Delta H_{\text {vap }}^{\infty}$ is the heat of vaporization of the bulk liquid and $N_{A}$ is Avogadro's number, less the amount of work required to increase the surface of the cluster. The work term is given by the product of the surface tension and the incremental increase in area, $\sigma d A_{g} / d g$,

$$
q_{g}=h_{\text {vap }}^{\infty}-\sigma \frac{d A_{g}}{d g}=h_{\text {vap }}^{\infty}\left(1-\frac{\sigma A_{1}}{h_{\text {vap }}^{\infty}} g^{-0.33}\right) .
$$

The heat of vaporization per molecule is quite similar for a range of materials commonly studied in nucleation experiments and at $298 \mathrm{~K}, h_{\text {vap }}^{\infty}$ for water is $\approx 7.3$ $\times 10^{-13} \mathrm{erg} / \mathrm{molecule}(\approx 20 \mathrm{kT})$, while the normal alcohols range from $6.2 \times 10^{-13} \mathrm{erg} / \mathrm{molecule}$ for methanol to $10.2 \times 10^{-13} \mathrm{erg} / \mathrm{molecule}$ for hexanol. ${ }^{12}$ A typical value for $\sigma A_{1} / h_{\text {vap }}^{\infty}$ for water is approximately 0.5 for $T=273$ to $323 \mathrm{~K}$, decreasing only slightly as $T$ increases, and thus for small $g, q_{g}$ is reduced significantly over the value of $h_{\text {vap }}^{\infty}$.

An alternate expression for the energy added per impinging molecule, $q_{g}$, is given by the empirical correlation developed by Freund and Bauer ${ }^{13}$ who considered the normalized condensation energy per molecule $\left(\Delta E_{g} / g\right) / \Delta E^{\infty}$, where $\Delta E_{g}$ is the energy of the reaction $X_{g} \rightarrow g X$. Using five sets of theoretical data and one set of experimental data a least squares fitting of the data to the functional form $\Delta E_{g} / g=\Delta E^{\infty}\left(1-a g^{-b}\right)$ gave

$$
\frac{\Delta E_{g}}{g}=\Delta E^{\infty}\left(1-g^{-0.25}\right) \text {. }
$$

By assuming $\Delta E_{g}$ is approximately equal to $\Delta H_{g}$ (for water at $298 \mathrm{~K} \Delta E^{\infty} \cong 0.95 \times \Delta H^{\infty}$ ) this gives

$$
q_{g}=h_{\text {vap }}^{\infty}\left(1-0.75 g^{-0.25}\right) \text {. }
$$

The similarity of the two equations both in form and in the magnitude of the coefficients suggests that the choice of which equation is used in the energy balances will not affect the general results, although for a given set of conditions the final nucleation rates will differ. We will use the correlation proposed by Freund and Bauer because it is based on a large number of calculations for small clusters for which the liquid drop model implicit in Eq. (10) is more difficult to justify.

A change in the temperature of a cluster from $T_{\mathrm{amb}}$ will necessarily alter the rate at which monomers leave the cluster. Applying Eq. (4) at two different temperatures gives the following correction to the isothermal evaporation rate in terms of the cluster temperature $T_{g+1}$ and the ambient temperature $T_{\text {amb }}$ for small temperature differences or when $\sigma$ is not a strong function of $T$ :

$$
\begin{aligned}
\alpha_{g+1}\left(T_{g+1}\right)= & \alpha_{g+1}\left(T_{\mathrm{amb}}\right) \exp \left[( 1 - \frac { T _ { \mathrm { amb } } } { T _ { g + 1 } } ) \left\{\frac{h}{k T_{\mathrm{amb}}}\right.\right. \\
& \left.\left.-\frac{\sigma A_{1}}{k T_{\mathrm{amb}}}\left[(g+1)^{2 / 3}-g^{2 / 3}\right]\right\}+\frac{1}{2} \ln \frac{T_{\mathrm{amb}}}{T_{g+1}}\right] .
\end{aligned}
$$

If $\sigma$ is a strong function of temperature, Eq. (13) should be modified to read

$$
\begin{aligned}
\alpha_{g+1}\left(T_{g+1}\right)= & \alpha_{g+1}\left(T_{\mathrm{amb}}\right) \exp \left\{\left(1-\frac{T_{\mathrm{amb}}}{T_{g+1}}\right) \frac{h}{k T_{\mathrm{amb}}}\right. \\
& +\left[\frac{\sigma\left(T_{\mathrm{amb}}\right)}{T_{\mathrm{amb}}}=\frac{\sigma\left(T_{g+1}\right)}{T_{g+1}}\right] \frac{A_{1}}{k} \\
& \left.\times\left[(g+1)^{2 / 3}-g^{2 / 3}\right]+\frac{1}{2} \ln \frac{T_{\mathrm{amb}}}{T_{g+1}}\right\} .
\end{aligned}
$$

In the simulations that follow, Eq. (14) was used to correct the evaporation rate for the difference in temperature between the clusters and the background gas.

\section{Rate of energy transfer by nonaccommodating molecules, $\boldsymbol{Q}_{\boldsymbol{g}}$}

The energy removed from a cluster by molecules that impinge but are not incorporated into the growing cluster is given by $Q_{g}$. For clusters large enough to be treated as droplets, but still small compared to the mean free path of the gas, this term should reduce to the expression proposed by Kantrowitz from kinetic gas theory,

$$
\begin{aligned}
Q_{g}= & A_{g} f_{g}\left[\beta_{\text {gas }} c_{v g a s}^{\prime} \alpha_{h g a s}+\left(1-\alpha_{m v a p}\right) \beta_{\text {vap }} c_{v \text { vap }}^{\prime} \alpha_{h v a p}\right] \\
& \times\left(T_{g}-T_{\text {amb }}\right),
\end{aligned}
$$

where $\beta_{\text {gas }}, c_{\text {vgas }}^{\prime}$, and $\alpha_{\text {hgas }}$ are the impingement rate, heat capacity per molecule, and thermal accommodation coefficient of the noncondensing gas molecules, $\beta_{\text {vap }}, c_{\text {vvap }}^{\prime}$, and $\alpha_{h v a p}$ are the impingement rate, heat capacity per molecule, and thermal accommodation coefficient of the vapor molecules, and $\alpha_{\text {mvap }}$ is the mass accommodation coefficient of the condensing vapor. Here $c_{v}^{\prime}=c_{v}+k T / 2$, where the additional energy $k T / 2$ appears because the molecules found impinging onto the surface represent a subset of the gas molecules that have velocities directed only toward the cluster and thus averaging only over this subset gives a higher energy than averaging over a random set of molecules in the vapor phase. ${ }^{5,6}$

Despite its convenient form, there are some difficulties in the straightforward application of Eq. (15) to describc the energy transfer over the entire range of cluster sizes $g$. This equation assumes that the energy transfer between a gas molecule and a liquidlike cluster is independent of the relative sizes of the two species and is only of the order of $c_{v} \Delta T \approx 10^{-16} \times \Delta T$ erg molecule ${ }^{-1}$ per collision. This assumption may not be appropriate for small $g$, where the molecular clusters and the impinging molecules are not terribly different in size. In this case it is reasonable to expect energy transfer to be similar to that which is observed experimentally ${ }^{14,15}$ or predicted theoretically ${ }^{16,17}$ during unimolecular reactions. Here a single collision between a highly excited molecule and a background gas molecule can transfer an average energy on the order of $\langle\Delta E\rangle=0.1-30 \mathrm{kcal} \mathrm{mol}^{-1}\left(=7 \times 10^{-15}-2 \times 10^{-12} \mathrm{erg} /\right.$ molecule) per collision. ${ }^{18}$ The amount of energy transferred per collision depends mainly on the relative sizes (number of atoms in the molecule) of the excited molecule and background gas and on the absolute energy of the 
TABLE I. Physical properties of water and air.

\begin{tabular}{|c|c|c|}
\hline$c_{\text {bwater vapor }}$ & $4.66 \times 10^{-16}$ & erg $\mathrm{K}^{-1}$ molecule ${ }^{-1}$ \\
\hline$c_{\text {pwater liquid }}$ & $1.26 \times 10^{-15}$ & $\operatorname{erg~} \mathrm{K}^{-1}$ molecule ${ }^{-1}$ \\
\hline$c_{\text {bair }}$ & $3.46 \times 10^{-16}$ & erg $\mathrm{K}^{-1}$ mqlecule ${ }^{-1}$ \\
\hline$h_{\text {vap }}^{\infty}$ & $9.29 \times 10^{-13}-6.67 \times 10^{-16} T$ & erg molecule ${ }^{-1}$ \\
\hline$\sigma$ & $120.88-0.167(T-273.15)$ & dyne $\mathrm{cm}^{-1}$ \\
\hline$\rho$ & 1.0 & $\mathrm{~g} \mathrm{~cm}^{-3}$ \\
\hline$m_{\text {water }}$ & $2.99 \times 10^{-23}$ & $\mathrm{~g}$ molecule ${ }^{-1}$ \\
\hline$m_{\text {air }}$ & $4.81 \times 10^{-23}$ & g molecule ${ }^{-1}$ \\
\hline
\end{tabular}

excited molecule, although the exact nature of the latter relationship is still rather controversial. ${ }^{17}$

These estimates of energy transfer demonstrate that if small clusters of the nucleating vapor are considered analogous to polyatomic molecules, then energy transfer by collisions with the background gas may be much higher than predicted by Eq. (15) in its current form and, furthermore, the amount transferred should be a function of g. For clusters composed of strongly interacting fragments, such as ionic species, the assumption of a polyatomic molecule is a good one ${ }^{5}$ and for water, or other highly hydrogen bonded molecular clusters, this assumption is probably still quite reasonable. Using the value for $h_{\text {vap }}^{\infty}$ for water from Table I, each condensing water molecule brings in about $7 \times 10^{-13} \mathrm{erg}$; thus if each collision with a background gas molecule is capable of removing up to $10^{-13}$ to $10^{-12}$ erg, ${ }^{18}$ temperature differences between the background gas and the smallest clusters should stay small at steady state even if the amount of background gas is reduced substantially below $1 \mathrm{~atm}$. To determine the true energy transfer between energetic water clusters and a cool background gas, calculations analogous to those performed by Bruehl and Schatz ${ }^{17}$ for energy transfer from an excited $\mathrm{CS}_{2}$ molecule to cool $\mathrm{He}$ gas molecules are required but such calculations are not presently available in the literature.

To predict the rate of energy transfer for small clusters, we will use some general results derived by Troe ${ }^{16}$ for the probability, $P\left(E^{\prime}, E\right)$, of an excited molecule with initial energy $E^{\prime}$ having a final energy $E$ after collision with a background gas molecule. These calculations assume that the excited molecule and the background gas form a collision complex in which total momentum and angular momentum are conserved during the collision process and that the species do not have strong chemical interactions. The values of $P\left(E^{\prime}, E\right)$ depend on the number of atoms in the excited molecule, $N_{a}$, and the number of atoms in the background gas molecule, $N_{m}$, in the expected manner, i.e., as $N_{a}$ increases for fixed $N_{m}$ the average energy transferred per collision with a background molecule decreases. Similarly for fixed $N_{a}$, the energy transfer per collision with a background molecule increases as $N_{m}$ increases. Table II gives the values of the average energy transferred per collision as a function of $N_{a}$ and $N_{m}$ assuming that $\langle\Delta E / k T\rangle$ is close to the peak in each of the probability transition curves. Figure 1 illustrates the variation of $\langle\Delta E / k T\rangle$ as a function of the number of triatomic molecules in the cluster $g=N_{a} / 3$ that has been extrapolated from the calcu-
TABLE II. Theoretical estimates of the average energy transferred between an excited molecule containing $N_{a}$ atoms and a background gas molecule containing $N_{m}$ atoms (Ref. 17).

\begin{tabular}{ccc}
\hline$N_{a}$ & $N_{m}$ & $\frac{\langle\Delta E\rangle}{k T}$ \\
\hline 15 & 1 & 2 \\
& 3 & 7 \\
& 5 & 11 \\
& 8 & 15 \\
3 & 12 & 20 \\
6 & 1 & 40 \\
9 & & 15 \\
15 & & 8 \\
30 & & 3.5 \\
\hline
\end{tabular}

lated data of Troe. ${ }^{16}$ In addition, the dashed line represents the energy transfer predicted by Eq. (15) per collision of a cluster with air when $c_{v}^{\prime}=4 \times 10^{-16}$ and assuming $\Delta T$ $=1 K$.

To maintain the convenient functional form of Eq. (15), that is $Q_{g} \propto\left(T_{g}-T_{\text {anb }}\right)$, the functional form of $\langle\Delta E / k T\rangle$ is used to define an effective energy transfer coefficient, $\phi(g)$ erg molecule ${ }^{-1} \mathrm{~K}^{-1}$, for the impinging molecules striking small clusters,

$$
\phi(g) \propto\langle\Delta E\rangle,=C g^{-1.3},
$$

where $C$ is an undetermined constant referred to as the energy transfer parameter. The lack of information regarding energy transfer to small clusters makes it impossible to independently assign a value to $C$ at this time. The expression for $Q_{g}$ for small clusters then becomes

$$
\begin{aligned}
Q_{g}= & A_{g} f_{g}\left[\beta_{\text {gas }} \phi_{\text {gas }}(g)+\left(1-\alpha_{m v a p}\right) \beta_{\text {vap }} \phi_{\text {vap }}(g)\right] \\
& \times\left(T_{g}-T_{\text {amb }}\right),
\end{aligned}
$$

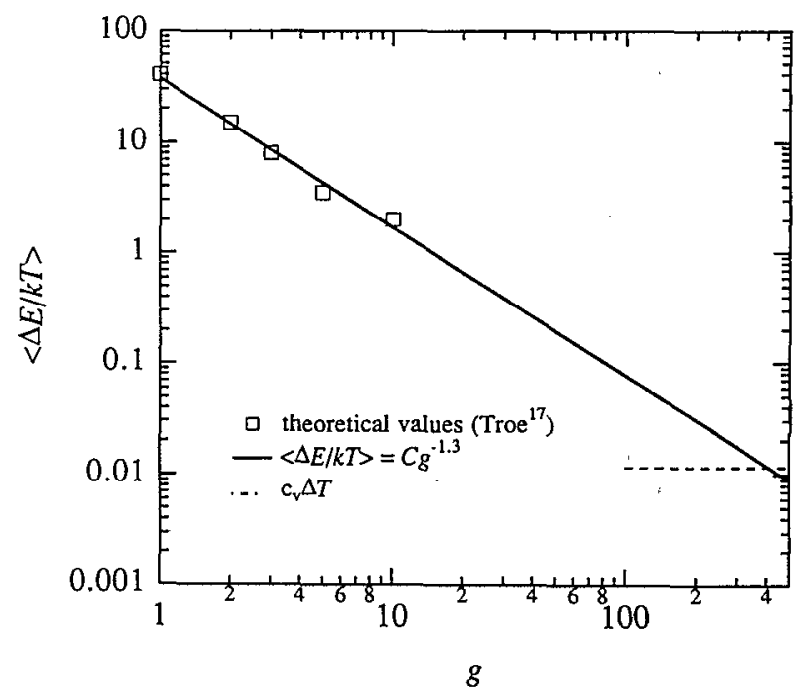

FIG. 1. Average energy transferred in a collision between a monatomic gas and a cluster containing $g$ triatomic molecules. The squares are the results of simulations performed by Troe (Ref. 17). 
where $\phi_{\text {gas }}$ and $\phi_{\text {vap }}$ are the energy transfer coefficients of the inert gas and nonaccommodated vapor, respectively. Inserting the appropriate expressions for $\phi_{\text {gas }}$ and $\phi_{\text {vap }}$ gives the more explicit functional dependence of $Q_{g}$ on $g$ for the small clusters,

$$
\begin{aligned}
Q_{g}= & A_{g} f_{g}\left[\beta_{\text {gas }} C_{\text {gasg }} g^{-1.3}+\left(1-\alpha_{m v a p}\right) \beta_{\text {vap }} C_{\text {vap }} g^{-1.3}\right] \\
& \times\left(T_{g}-T_{\text {amb }}\right) .
\end{aligned}
$$

Although from Fig. 1 it is clear that for sufficiently large $g$ Eq. (18) should become independent of $g$ to match the values of Eq. (15), these values of $g$ are larger than the largest cluster size that will be considered in the simulations presented here. Therefore, Eq. (18) is the form of the energy transfer equation used in all of the simulations.

In the modeling of experimental results for the nucleation of iron vapor, Freund and Bauer ${ }^{13}$ found it necessary to use an adjustable heat transfer coefficient $\theta=h^{*}(g$ $+1)^{-1}$ to reproduce the observed time variation in the enthalpy of their system and commented that with the values of $h^{*}$ found to provide the best fit, energy transfers for the smallest cluster were consistent with observations from unimolecular reaction theory. The functional form chosen here is very similar to that of Freund and Bauer, ${ }^{13}$ $g^{-1.3}$ vs $(g+1)^{-1}$, but by introducing the results from unimolecular reaction theory we have demonstrated the origin of this functional form and illustrated why it is appropriate. It is also clear that as numerical simulations become available that in essence combine the approaches of Bruehl and Schatz ${ }^{17}$ and Plummer and Chen, ${ }^{4}$ a more realistic model of energy transfer between clusters and a noncondensing gas could be developed. This is certainly one avenue for incorporating the chemical nature of both the nucleating and inert species into the general formalism of nucleation theory.

\section{NONISOTHERMAL NUCLEATION RATES}

The strong coupling between the mass and energy equations should result in more complex behavior of the nucleating system than is observed under the assumption of isothermal nucleation, especially in the transient during the approach to steady state. To investigate this behavior Eqs. (1) and (9) are solved simultaneously for various levels of saturation and energy transfer rates. The model system investigated is the nucleation of liquid water vapor from the supersaturated vapor phase at $263.2 \mathrm{~K}$, because the isothermal relaxation to steady state of this system has been studied previously ${ }^{10,11}$ and physical properties of water are readily available.

Before solving the cluster concentration and energy equations simultaneously, the population equations were solved isothermally for various initial conditions and boundary values of $g_{\min }$ and $g_{\max }$. In both the isothermal and nonisothermal simulations the smallest cluster considered, $g_{\min }$ is assumed to be at its equilibrium concentration and at the ambient temperature at the start of the simulation and to remain at these values throughout the simulation. In addition, the monomer concentration is assumed to remain constant and the monomer is at the ambient

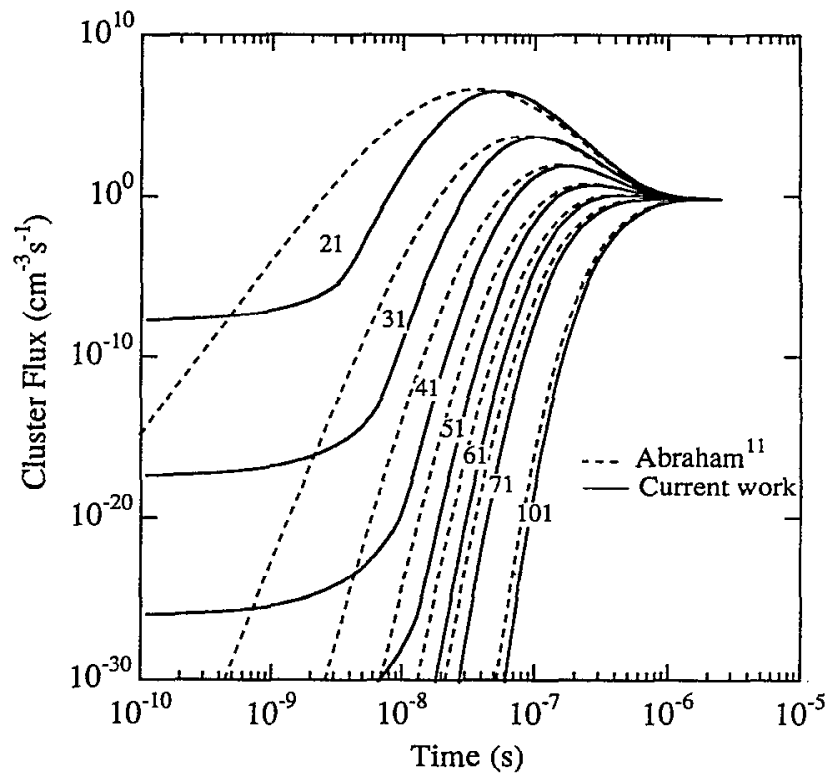

FIG. 2. The evolution of the cluster flux with time of selected clusters for water at $T_{\text {amb }}=263.2 \mathrm{~K}$ and in response to a sudden increase of $S$ to $S=4.91$. The final steady state is not affected by the choice of initial conditions, $S=0$ or 1.0 , or the number of clusters evolved. Isothermal conditions are assumed to hold.

temperature. Rather than applying the Szilard boundary condition, $f_{g \max +1}=0$, where $g_{\max }$ is the largest cluster, an extrapolated value for $f_{g \max +1}$ is used, based on the values of $f_{g \max }$ and $f_{g \max -1}$. This choice gives a slightly smoother transition for the right hand boundary condition and avoids some of the numerical stability problems associated with setting $f_{g \max +1}=0$.

Results identical to those of Abraham ${ }^{10}$ were obtained for identical initial conditions and physical property values. From the results in Fig. 2 it is clear that during the approach to steady state, mass fluxes through the subcritical clusters can exceed the final steady state flux by many orders of magnitude. This overshoot may be understood in light of the following observations. As the saturation level, $S$, is suddenly increased from its initial value $S_{0}$ to a higher value $S_{1}$, the forward mass flux immediately increases by a factor of $S_{1} / S_{0}$, while the backward mass flux remains a function of the evaporation rate, which depends only on the properties of the cluster and not the saturation level, and the cluster concentration. Furthermore since the concentration of subcritical clusters at steady state, $f_{g}$, is close to its cquilibrium value, $N_{g}^{e}$ the ratio of adjacent steady state cluster concentrations determined by $S_{0}, f_{g} / f_{g+1}$, is larger than the equivalent ratio at $S_{1}$ because $N_{g}^{e} / N_{g+1}^{e}$ $\propto S^{-1}$. Finally, because clusters grow only by the addition of monomer, the number of clusters of size $g+1$ can only increase after the number of clusters of size $g$ and $f_{g}$ will reach its new steady state value before $f_{g+1}$. Thus there will be some time period for which $f_{g} / f_{g+1}$ is larger than its final steady state ratio at $S_{1}$ and yet $f_{g} \cong f_{g}\left(S_{1}\right)$ leading to the observed overshoot. As $f_{g} / f_{g+1}$ relaxes to its steady state value, so does the net mass flux. It is interesting to 
examine whether similar transient behavior is exhibited by the average energy of the clusters before they achieve their final steady state, how large these transients are and how they can affect the approach to steady state and the final steady state nucleation rate.

As illustrated in Fig. 2, setting the values of $g_{\min }$ $=2, g_{\max }=200$ and $f_{g}^{0}$ equal to the equilibrium distribution at $S_{0}=1$ from Abraham's values of $g_{\min }=10, g_{\max }=100$, and $f_{g}^{0}=0$ for $g>g_{\min }$, did not affect the steady state values of cluster concentration, nucleation rate (steady state current), $J$, or time lag, $\tau$. Only the times at which the smaller cluster concentrations began to increase changed. This discrepancy occurs because Abraham assumed $f_{10}$ was at its equilibrium value throughout the simulation, while with $g_{\min }=2$ our simulation requires a finite amount of time before $f_{10}$ is essentially at its equilibrium value. The equations were not solved with $g_{\min }=1$ because of the large increase in computing time incurred from that for $g_{\min }$ $=2$. Since the dimer concentration essentially reached its equilibrium value in the first timestep, $<10^{-10} \mathrm{~s}$, setting $g_{\min }=2$ was justified. Evolving 150 clusters was adequate except in cases where $g^{*}$ was larger than about 90, in which case $g_{\max }=200$ was used. As expected, including a mass accommodation coefficient, $\alpha_{m \text { vap }}<1$, simply reduced the final nucleation rate $J$ to $J=\alpha_{\text {mvap }} J_{0}$ and increased the time required to reach steady state, $\tau$, to $\tau=\tau_{0} / \alpha_{\text {mvap }}$.

The correct value of $\alpha_{m \text { vap }}$ for water is still a matter of controversy. ${ }^{19}$ Based on the limited set of simulations with clusters containing both 5 and 20 molecules, Plummer and Chen ${ }^{4}$ found that collision between a monomer and the cluster always resulted in the absorption of the monomer by the cluster. No scattering or evaporation events were observed and thus, at least for the temperature range investigated, $\leqslant 93 \mathrm{~K}$, these simulations suggest that for water an accommodation coefficient close to one is a reasonable assumption. However, the authors also note that at higher initial temperatures the increase in temperature due to incorporation of the monomer may lead to metastable structures and subsequent evaporation of a monomer. If this absorption-evaporation sequence occurs rapidly enough the net effect would be the same as an accommodation coefficient less than one that depends on the initial temperature of the cluster.

If the mass accommodation coefficient for the condensing vapor is assumed to be 1 , the sole mechanism available for efficient cooling of clusters is collision with the incrt background gas. Because the value of the energy transfer parameter $C$ is not readily available and only appears in combination with the gas collision rate $\beta_{\text {gas }}$, the combination of $\beta_{\text {gas }} C$ will be varied as a single adjustable parameter in these simulations. Figure 3 illustrates the effects of varying the parameter $C^{*}=C \beta_{\text {gas }}$ from $C^{*}=C_{0}^{*}=4 \times 10^{-11}$ erg cm $\mathrm{cm}^{-2} \mathrm{~s}^{-1} \mathrm{~K}^{-1}$ to $C^{*}=0.0 \mathrm{erg} \mathrm{cm}^{-2} \mathrm{~s}^{-1} \mathrm{~K}^{-1}$, with the results of the isothermal nucleation calculations included for comparison. The effect of decreasing $C^{*}$ becomes significant for $C^{*} \approx 0.001 C_{0}^{*}$, but even for $C^{*}=0.0$, i.e., no background gas, the nucleation rate is decreased only by about 3 orders of magnitude. Varying the value of $C^{*}$ can be interpreted physically as varying the pressurc of back-

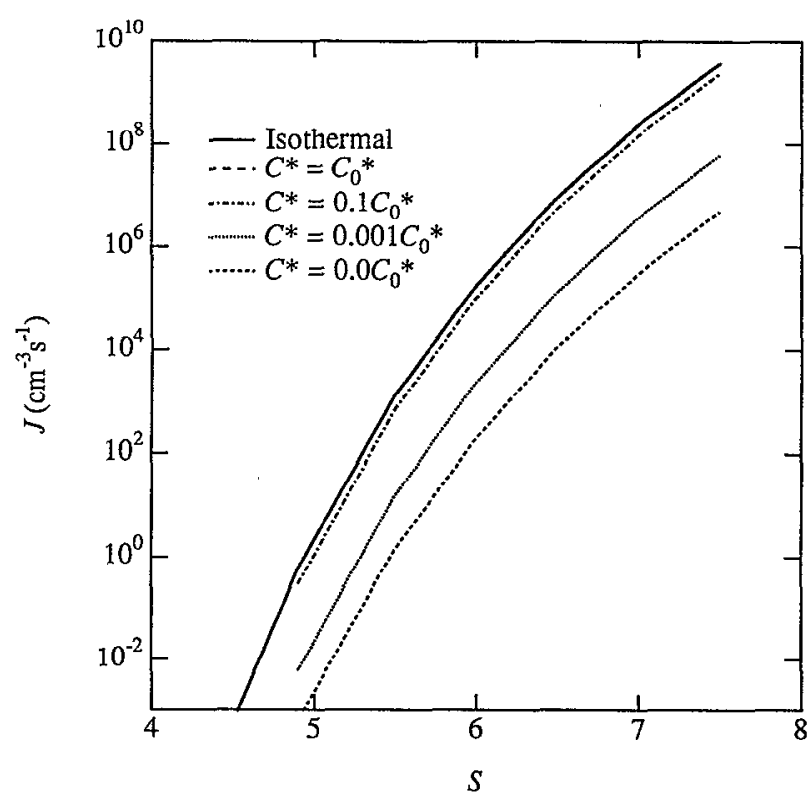

FIG. 3. Homogeneous nucleation rate of water at $T_{\mathrm{amb}}=263.2 \mathrm{~K}$ as a function of the energy transfer parameter $C^{*}$.

ground gas available while maintaining the same saturation level of the nucleating species. Alternatively, if the amount of background gas is fixed, a decrease in $C^{*}$ corresponds to a decrease in the efficiency of energy transfer from the clusters to the background gas when, for example, a monatomic gas has been substituted for a larger molecule.

Figure 4 illustrates the temperature distribution of the clusters as a function of time for a value of $C^{*}=4$ $\times 10^{-11} \mathrm{erg} \mathrm{cm}^{-2} \mathrm{~s}^{-1} \mathrm{~K}^{-1}$ and for a sudden increase in $S$ from 1 to 4.91 . Clearly there is an energy transient that moves through the clusters as the new steady state cluster distribution is reached. The final temperature distribution, shown on an expanded scale in Fig. 5, is, however, very close to the ambient temperature $T_{\text {amb }}=263.2 \mathrm{~K}$, with significant deviations only near and above the critical cluster size. The significantly higher temperatures predicted for final clusters should be ignored as this is simply an effect of the proximity to the boundary. If clusters of size $g_{\max +1}$ are underestimated slightly, the number of clusters of size $g_{\max }$ that are formed by evaporation events is too low compared to those formed by condensation events and the average temperature is too high. Incrcasing the number of clusters that are evolved, as indicated by the solid line in Fig. 5, clearly demonstrates that this sharp jump is not a physical phenomenon. The critical nucleus, $g^{*}$ for these conditions is determined by searching for that value of $g$ at which $\beta_{g-1} / \alpha_{g}$ changes from less than 1 to greater than 1 . This corresponds to $g^{*}=74$, compared to $g^{*}=71$ under isothermal conditions and the increase in $g^{*}$ is accompanied by a corresponding increase in the time required to reach steady state.

As expected, particles growing past the critical point increase in temperature. This agrees with the observations of Feder et al..$^{6}$ However, in contrast to Feder et al. ${ }^{6}$ and Ford and Clement, ${ }^{9}$ we predict that the clusters on either 


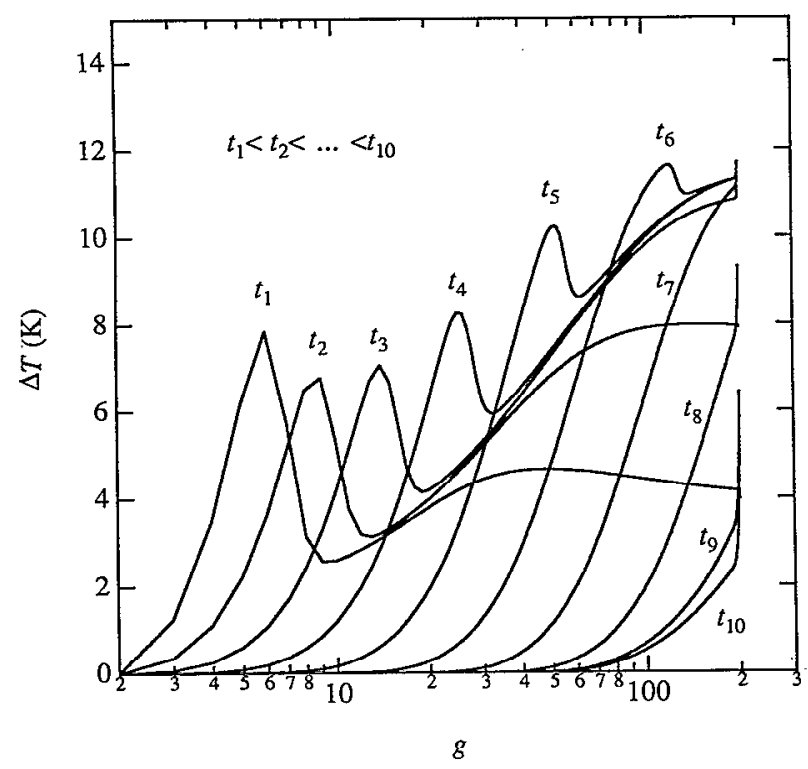

FIG. 4. Cluster temperature distribution as a function of time for water at $T_{\mathrm{amb}}=263.2 \mathrm{~K}$ with $C^{*}=C_{0}^{*}$ and in response to a sudden increase of $S$ from 1 to 4.91 . The values of time are $t_{1}=2.8 \times 10^{-10} \mathrm{~s}, t_{2}=7.6$ $\times 10^{-10} \mathrm{~s}, t_{3}=2.1 \times 10^{-9} \mathrm{~s}, t_{4}=5.7 \times 10^{-9} \mathrm{~s}, t_{5}=1.6 \times 10^{-8} \mathrm{~s}, t_{6}$ $=4.4 \times 10^{-8} \mathrm{~s}, t_{7}=1.2 \times 10^{-7} \mathrm{~s}, t_{8}=3.3 \times 10^{-7} \mathrm{~s}, t_{9}=9.1 \times 10^{-7} \mathrm{~s}$, and $t_{10}=2.5 \times 10^{-6} \mathrm{~s}$.

side of the critical cluster are on average warmer than the ambient temperature.

Looking at the time evolution of the temperature in particular cluster classes, Fig. 6, for the same conditions as in Figs. 4 and 5 and comparing these to the mass fluxes for the same clusters illustrated in Fig. 2, demonstrates that the peak in the temperature of a cluster class corresponds to the initial rapid increase in the concentration of that

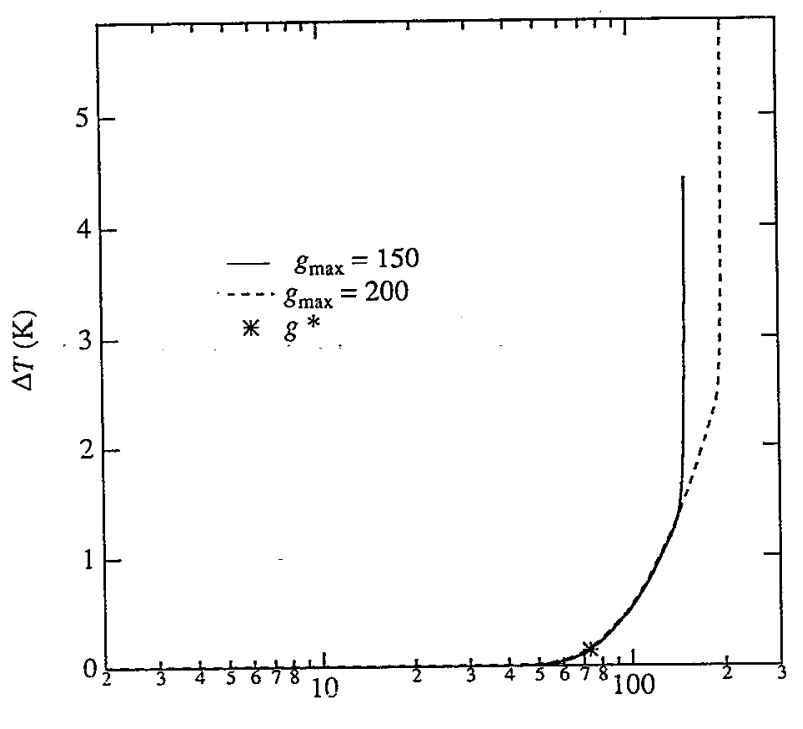

$g$

FIG. 5. The steady state cluster temperature distribution for $C^{*}=C_{0}^{*}$ and for $g_{\max }=150$ and 200 .

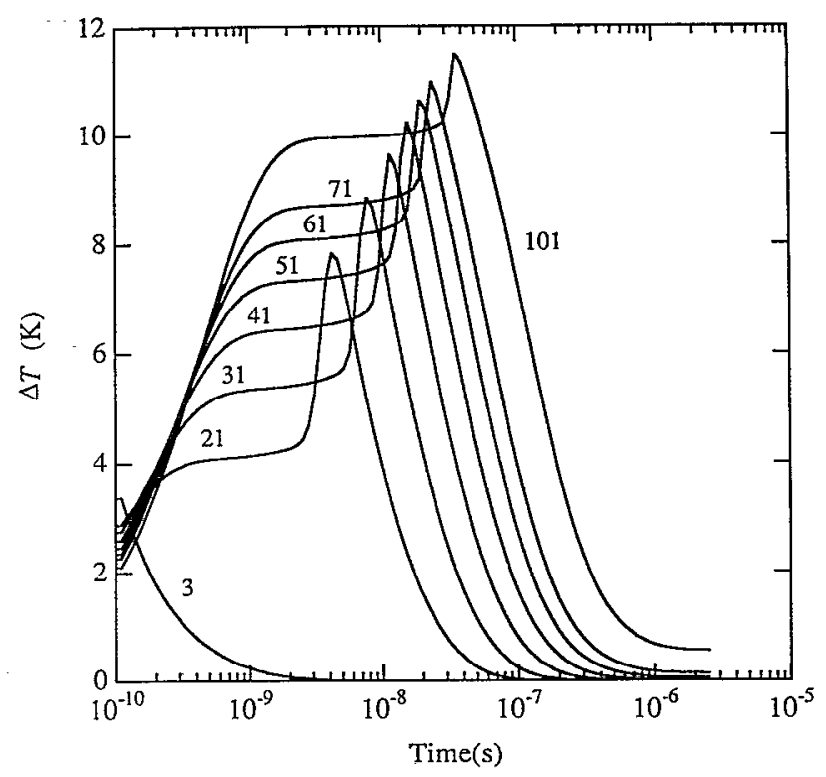

FIG. 6. Transient cluster temperature in the homogeneous nucleation of water at $T_{\text {amb }}=263.2 \mathrm{~K}$ with $C^{*}=C_{0}^{*}$ and in response to a sudden increase of $S$ from 1 to 4.91 .

cluster class. (Although Fig. 2 is strictly the result of an isothermal calculation, the high value of $C^{*}$ ensured that the cluster flux evolution in this case is very close to those in Fig. 2.) Physically this simply corresponds to the fact that suddenly far more clusters of that size are the result of condensation than evaporation and that the large mass flux is overwhelming the ability of the background gas to remove energy. It is interesting to observe the rather large transients in temperature that are predicted during the approach to steady state even when the value of $C^{*}$ is high enough to predict minimal impact on the final nucleation rate.

The results in Figs. 2 to 6 present the following physical picture of nonisothermal nucleation. When $q_{g}$ is positive, the nonisothermal nucleation rate is lower than the isothermal rate because the average temperature of the clusters in the critical region is higher than the ambient temperature, and thus the evaporation rate is higher than in isothermal nucleation. As a consequence of the higher evaporation rates the size of the critical cluster, $g^{*}$, increases and hence the time required to reach steady state increases. This increase in time to reach steady state associated with an increase in $g^{*}$ is analogous to that observed in isothermal nucleation as $S$ decreases.

When the value of the mass accommodation coefficient, $\alpha_{\text {mvap }}$ is varied in these calculations, competing effects are brought into play. As in isothermal nucleation, reducing $\alpha_{\text {mvap }}$ reduces the nucleation rate. However, in nonisothermal nucleation a lower value of $\alpha_{\text {mvap }}$ results in additional time available for energy transfer between incorporation of the condensing species. The impinging but noncondensing molecules also provide additional energy transfer and overall the deviation from isothermal nucleation rates should decrease as $\alpha_{\text {mvap }}$ decreases. Depending on the 


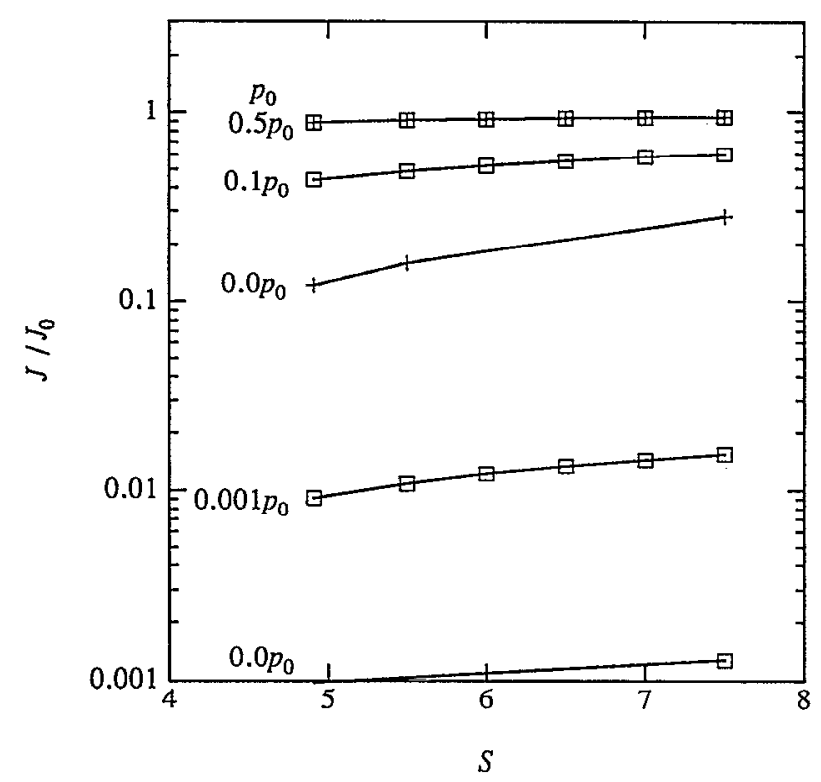

FIG. 7. Nonisothermal nucleation rates of water normalized with respect to the isothermal value, $J / J_{0}$, at $T_{\text {amb }}=263.2 \mathrm{~K}$ as a function of the background gas pressure for $\alpha_{\text {mvap }}=0.5$ and 1.0 and for fixed energy transfer parameter $C$.

relative energy transfer efficiencies, noncondensing vapor molecules may contribute proportionally more or less to the cooling than relative impingement rates would predict. For simplicity, in these simulations we have set $\phi_{\text {gas }}(g)$ $=\phi_{\text {vap }}(g)$ and thus cooling rates due to the two species will be proportional to their relative impingement rates.

A set of simulations similar to those summarized in Fig. 3 were conducted using a mass accommodation coefficient, $\alpha_{\text {mvap }}=0.5$. In order to compare the two sets of results, the normalized nucleation rates, $J / J_{0}$, where $J_{0}$ is the isothermal nucleation rate, are presented in Fig. 7 as a function of the saturation level for $\alpha_{\text {mvap }}=1.0$ (squares) and $\alpha_{m \text { vap }}=0.5$ (crosses). In the discussion that follows the separate curves are interpreted as simulations corresponding physically to levels of background gas varying from $p_{0}$ to 0.0 with a constant value of the energy transfer parameter $C_{\text {gas }}=C_{\text {vap }}=C$. The effect of the mass accommodation coefficient is apparent when comparing the curves for $\alpha_{m \text { vap }}=0.5$ and 1.0 at the highest background gas pressure $p_{0}$. As expected the deviation from the isothermal case is less for $\alpha_{\text {mvap }}=0.5$ than for $\alpha_{\text {mvap }}=1.0$ and this difference is best understood by realizing that normalizing Eqs. (1) and (9) by dividing by $\alpha_{\text {mvap }}$ results in an overall energy transfer coefficient proportional to

$$
C^{*}=\frac{\beta_{\mathrm{gas}} C_{\mathrm{gas}} g^{-1.3}+\left(1-\alpha_{\text {mvap }}\right) \beta_{\mathrm{vap}} C_{\mathrm{vap}} g^{-1.3}}{\alpha_{\text {mvap }}} .
$$

Thus at high levels of background gas where the contribution to cooling from nonaccommodated molecules is insignificant we would expect the same degree of deviation from isothermal nucleation for $\alpha_{m \text { vap }}=0.5$ and $p=0.5 p_{0}$ as for $\alpha_{m \text { vap }}=1.0$ and a background pressure of $p=p_{0}$ as these sets of conditions correspond to the same value of $C^{*}$.

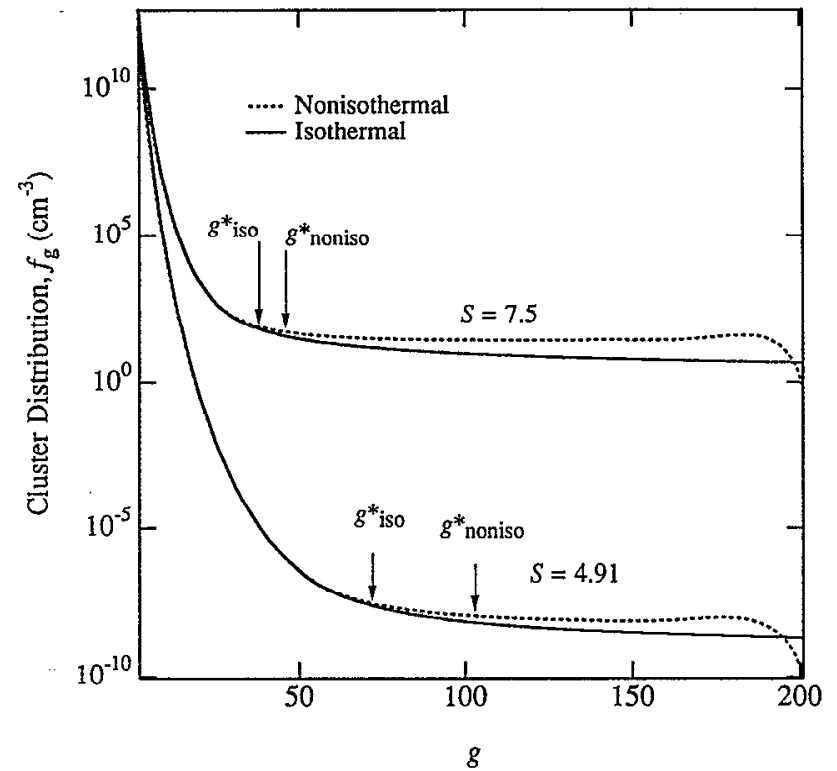

FIG. 8. Steady state cluster distributions for isothermal and nonisothermal nucleation at two saturation levels and $\alpha_{\text {mvap }}=0.5$ illustrate the shift in the critical nucleus due to increased evaporation rates in the critical region.

Figure 7 demonstrates that this is indeed the case. With the background gas removed the effect of cooling by the nonaccommodating vapor molecules becomes important and the departure from the nonisothermal results is less severe for the case of $\alpha_{\text {map }}=0.5$ simply because here the nonaccommodated molecules are providing the only means of energy transfer. Simulations were done which considered the possibility of radiative cooling of the particles, but for water at these temperatures this had no effect.

The effect of nonisothermal conditions on the steady state distribution of clusters is also interesting. Figure 8 compares the isothermal and nonisothermal cluster distributions for simulations in which $\alpha_{\text {mvap }}=0.5$ and the background gas pressure $p_{0}=0.0$. The increase in the size of the critical nucleus for the nonisothermal case realtive to the isothermal calculation is clearly illustrated. Although for a given value of $g$ the concentration of clusters is greater in the nonisothermal than in the isothermal case, the critical cluster concentration $f_{g^{*}}$ is lower under nonisothermal conditions and $I / J_{0}=f_{g^{*}} / f_{g^{*}, 0}$. The sharp drop in the cluster concentrations for the nonisothermal calculation at the largest clusters is again a consequence of the proximity to the boundary and reflects the extremely rapid increase in temperature observed in this region in Fig. 5.

Although the calculations presented here involve water as the model system, there is nothing in the general analysis that prevents this approach from being applied to other species. The key issues remain the uncertainty in using a single temperature and evaporation coefficient to characterize the behavior of a single cluster class and the need for a better understanding of energy transfer processes to small clusters. The two parameters that determine the importance of nonisothermal effects in gas-phase nucleation are the latent heat of vaporization and the rate of 
energy transfer for small clusters. The former is reasonably well characterized and does not vary greatly for the species that are of common interest; the latter represents a poorly understood phenomenon that warrants further investigation.

\section{SUMMARY AND CONCLUSIONS}

The phase transition as a monomer condenses onto or evaporates from a cluster is necessarily accompanied by a change in energy. In this paper cluster mass and energy balances are solved simultaneously to determine the effect including such an energy balance has on both the final nucleation rate and the approach to steady state. The energy balance assumes that the final temperature of the clusters in a given class is determined by a balance between the energy due to cluster growth and evaporation and energy transfer by collision with inert background gas molecules. The collisional energy transfer is described using a model based on energy transfer in unimolecular reaction theory.

The steady state nonisothermal nucleation rate is found to be a strong function of the degree of cooling; in all cases including an energy balance led to an increased average temperature of the clusters and a decreased final nucleation rate. During the approach to steady state, temperature transients much larger than the final steady state temperature deviation were observed and were associated with the onset of significant mass flux through a cluster class. The increased cluster temperatures and associated higher evaporation rates also led to an increase in $g^{*}$ and hence an increase in the time required to establish steady state nucleation. The effect of a mass accommodation coefficient less than 1.0 reduces the deviation of the final nucleation rate from the isothermal rate both by providing extra time for temperature equilibration to occur by collision with background molecules between incorporation of vapor molecules and by providing additional energy transfer by molecules that impinge but are not incorporated. The latter effect is important for low background gas pressures. This work also shows how the nature of the background gas can play a specific role in the nucleation process by changing the rate at which energy is transferred from the growing cluster.

\section{ACKNOWLEDGMENTS}

This work was supported by National Science Foundation Grant No. ATM-9003186 and by the Alberta Heritage Scholarship Fund (BEW). The authors wish to acknowledge helpful discussions with Frank G. Shi.

1 F. F. Abraham, Homogeneous Nucleation Theory (Academic, New York, 1974).

${ }^{2}$ A. C. Zettlemoyer, Nucleation (Dekker, New York, 1969).

${ }^{3}$ R. T. V. Kung and S. H. Bauer, Shock Tube Research, 8th International Symposium, Chapman and Hall, London, 1971, Paper No. 61.

${ }^{4}$ P. L. M. Plummer and T. S. Chen, J. Chem. Phys. 86, 7149 (1987).

${ }^{5}$ A. Kantrowitz, J. Chem. Phys. 19, 1097 (1951).

${ }^{6} \mathrm{~J}$. Feder, K. C. Russel, J. Lothe, and G. M. Pound, Adv. Phys. 5, 111 (1966).

${ }^{7}$ E. E. Salpeter, J. Chem. Phys. 58, 4331 (1973).

${ }^{8}$ A. P. Grinin and F. M. Kuni, Theor. Math. 80, 968 (1989).

${ }^{9}$ I. J. Ford and C. F. Clement, J. Phys. A 22, 4007 (1989).

${ }^{10}$ F. F. Abraham, J. Chem. Phys. 51, 1632 (1969).

${ }^{11}$ W. G. Courtney, J. Chem. Phys. 36, 2009 (1962).

${ }^{12}$ Enthalpies of Vaporization of Organic Compounds, IUPAC, Chem Data Series No. 32.

${ }^{13}$ H. J. Freund and S. H. Bauer, J. Phys. Chem. 81, 994 (1977).

${ }^{14}$ H. Hippler, J. Troe, and H. J. Wendelken, J. Chem. Phys. 78, 6709 (1983).

${ }^{15}$ D. C. Tardy and B. S. Rabinovitch, J. Chem. Phys. 48, 1282 (1968).

${ }^{16}$ J. Troe, Ber. Bunsenges. 77, 665 (1973).

${ }^{17}$ M. Bruehl and G. Schatz, J. Chem. Phys. 89, 770 (1988).

${ }^{18}$ See also Table '10.1 in P. J. Robinson and K. A. Holbrook, Unimolecular Reactions (Wiley-Interscience, London, 1972).

${ }^{19} \mathrm{H}$. K. Cammenga, in Current Topics in Material Science, edited by $\mathrm{E}$. Kaldis (North-Holland, Washington, D.C., 1980), Vol. 5, p. 335. 